\section{On homo loquens} Paul Fletcher

Uniquely Human: The Evolution of Speech, Thought and Selfless Behavior. By Philip Lieberman. Harvard University Press: 1991. Pp.210. \$27.95, $£ 22.25$.

Chimpanzees cannot talk. This indisputable fact is dealt with by linguists in different ways. The inability of species closely related to humans either to speak, or to learn a linguistic system of appropriate complexity, but expressed through some other medium (for example, manual signs) is for the (majority) chomskyan school a matter of some significance. It seems to confirm the distinct and unique status of humans and their language $v i s$ - $a$-vis our evolutionarily close relatives and their communication systems. Once a dichotomy between human language and animal communication is accepted, the research task for the chomskyan linguist (and, it has to be said, most others) is then the investigation of the principles of organization of human language in general and languages in particular. This research proceeds without reference to the evolution or present state of the anatomical structures or brain mechanisms which support these principles. Indeed, except in studies of language breakdown in adults, particularly adult aphasia, it is fair to say that brain-behaviour relationships are not of direct interest to many linguists.

There is however a menshevik tendency, of which Philip Lieberman has been a leading light, whose response to the uniquely human gift of tongues is to try to understand how and when the discontinuity between modern man and his predecessors came about. A major part of Uniquely Human reviews more than two decades of his research on this and related issues. Starting from the end-point - the current anatomy and physiology of the human speech-production apparatus - Lieberman calls into aid the comparative anatomy of the vocal mechanisms of extant primates, and interrogates the hominid fossil record. Crucial differences emerge between modern man, and his relatives and ancestors, in the shape of the supralaryngeal vocal tract and the position of the larynx. Because we are aware that chimpanzees do not talk, we can take it, or so the argument goes, that any archaic hominid whose laryngeal position and supralaryngeal vocal tract configuration resembles that of the modern chimpanzee could not talk either. And the inability to talk, in Lieberman's view, means the absence of those neural mechanisms that serve speech, and also the absence of syntax and the (related) neural mechanisms that serve it. So the nonhumanlike vocal tract of the La Chapelle-auxSaints neanderthal, buried about 50,000 years ago in southwestern France, suggests that if he did have speech, it was not recog- nizably human, and unlikely to be supported by the same brain mechanisms that support modern man's communication system.

This line of argument, attractive and intriguing as it may be, is not uncontroversial. Other researchers have probed Lieberman's chain of reasoning at various points. The conclusion that this particular neanderthal did not have speech rests on both a reconstruction of his vocal tract from basicranial measurements, and computer modelling of possible formant frequencies for vowels from this vocal tract. Both aspects of the investigation have been subject to detailed attacks, which in turn have provoked spirited ripostes. For a member of the linguistic majority, it is hard to see how the disputes can be resolved. In the absence of the critical data (which John Marshall once suggested, presumably not altogether seriously, would be

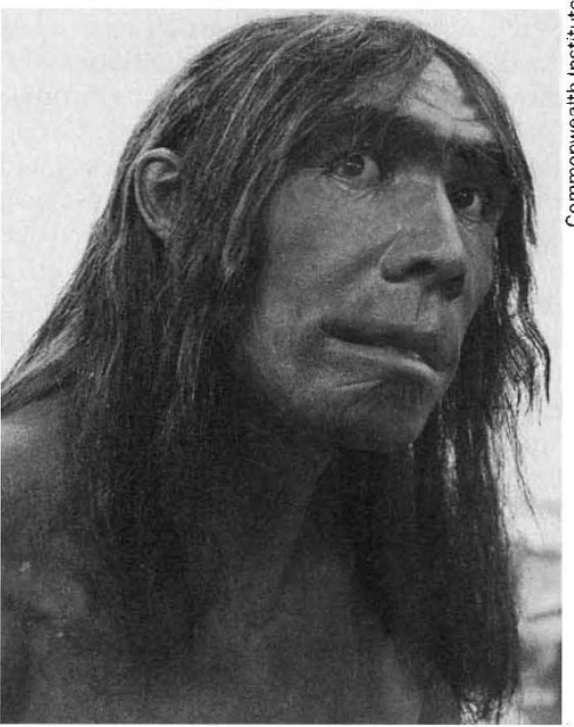

Could she speak? - life-size model of neanderthal female.

the discovery of a deep-frozen neanderthal who was susceptible to resuscitation) the comparative and evolutionary perspective insisted on by Lieberman would remain an interesting modern sidelight on speculations on the origin of language.

As it happens, this is not the end of the story. Lieberman's ideas on the representation, learning and breakdown of language in modern man, which are influenced by his evolutionary and comparative studies, are also provocative. For him, the availability of a human vocal tract guarantees important brain mechanisms. The brain has to be able to control the complex manoeuvres necessary for the control of the rapid articulatory movements of speech, which result in between 25 and 50 sounds being produced per second. In addition, the human perceptual apparatus has to be sophisticated enough to achieve resolution of the temporal and spectral characteristics of such an acoustic signal. So far, so good. The evolutionary advantage of a system that could voluntarily produce and perceive rapidly transmissible message is obvious. But linguists have long observed that there is a 'dual articulation' to the organ- ization of speech: sounds are lawfully arranged in sequences, but then the words which consist of sequences of sounds are constructed in sentences which have a quite distinct set of rules (syntax) for their arrangement. Lieberman's assertion that there is nothing mysterious about syntax will come as a surprise to anyone familiar with the linguistic literature of the last 25 years. Syntax may be simple, in the sense that all languages have it, and all children learn it, but then from a similar perspective so is vision, or any other cognitive skill. For the organism the acquisition of syntax may be relatively effortless; the hard labour is understanding how it happens.

Of course, Lieberman's assertion is to some extent rhetorical. Although the story that links the anatomical characteristics of human-like vocal tracts to specialized brain mechanisms for the complexities of the motor control of speech production, and for the perception of rapidly transmitted sounds, is plausible, it is not at all clear how syntax might fit in to the account. He finesses the problem by proposing that the evolutionary basis for the brain mechanisms that underlie human syntactic ability is rapid vocal communication. So those mechanisms that had to develop and be selected to support speech "may have provided the preadaptive basis for rule-governed syntax". To buttress this argument he is almost required to play down the complexity of syntax, and ignore the quite distinct patterns of organization of sound and syntax in languages.

More substantive support for his view comes from studies of language-disordered individuals in whom verbal comprehension is very poor, but speech and syntax are accurate, though their language is described as semantically empty. This type of dissociation reported in sufferers from William's syndrome and some other conditions which also involve a more general and severe mental retardation, appears to suggest an uncoupling of speech and syntax from the rest of the linguistic and cognitive system. But there is contrary evidence, from research on both language-impaired children and adult aphasics, with normal intellectual abilities, which shows dissociations between speech and syntactic ability.

Uniquely Human is an uneven but rewarding and well-written book. The specialist will see it as swimming against the tide, and the nonspecialist should be warned that the author's perspective on homo loquens is not wholly representative of his field. The project is an ambitious one, and bound to have flaws. But in forcefully reminding those of his colleagues who may have forgotten, that there is a biological basis for what makes us human, Lieberman provides an alternative perspective which merits serious debate.

Paul Fletcher is in the Department of Linguistic Science, The University of Reading, Whiteknights, POBox 218, Reading, RG6 2AA, UK. 